\title{
A Constitutive Model of Pseudo-Hyperelasticity for Description of Rubber-Like Materials
}

\author{
Stanisław Jemioło ${ }^{1, *}$ \\ ${ }^{1}$ WUT The Faculty of Civil Engineering, Al. Armii Ludowej 16, 00-637 Warszawa, Poland
}

\begin{abstract}
A consistent thermomechanical model applicable to pseudoelastic behaviour of rubber-like materials has been proposed. The model is based on the Naumann and Ihlemann's approach [1] and the concept of material stiffening given by Ogden and Roxburg [2,3]. The constitutive relationships of psedo-hyperelasticity are derived for incompressible isotropic materials. Futhermore, a specific model describing the Mullins effect was proposed. The homogeneous deformations are discussed and the obtained results are compared with Mullins' and Tobin's [4] experimental data. Good correlation between theoretical and experimental data has been observed.
\end{abstract}

\section{Introduction}

Rubber-like materials exhibit a highly nonlinear elastic behaviour but in cyclic loading the stress-softening phenomenon is observed [4]. This effect is now widely known as the Mullins effect [5]. Therefore, the rubber-like materials cannot have a strain-energy function in the hyperelastic sense [6]. However, in a cyclic loading and unloading the stress-strain relationship does not vary much with the strain rate. If the strain-rate effect is ignored altogether; then the loading curve and the unloading curve (they are unequal) can be separately treated as a uniquely defined stress-strain relationship, which is associated with a strain-energy function. Each of these curves is a pseudo-elastic curve. During successive reloading the material responds softer. This is achieved by scaling the stress with an appropriate softening function whose material parameters can be directly calculated from experimental data $[2,3,5,7,8]$. These pseudo-elastic models were also widely used in biomechanics [9-12] for constitutive modelling of isotropic and anisotropic soft tissue.

Recently, it was shown in the paper [1] that the softening function proposed in $[2,3]$ and [8] leads to thermomechanically consistent material models. The aim of this paper is to introduce a new phenomenological model for the Mullins effect based on the strain-energy function of isotropic, incompressible rubber-like materials [13,14] and the free energy potential given by Naumann and Ihlemann [1]. It is an alternative approach to Ogden and Roxburg [2,3] which enables a consistent deduction of pseudo-elasticity models by introducing a suitable free energy potential. The insertion into the Clausius-Duhem inequality directly yields the constitutive relationships for the stress tensor and the dissipation.

*Corresponding author: s.jemiolo@ili.pw.edu.pl 


\section{Basic assumptions and constitutive relationships of pseudo- hyperelasticity}

The Clausius-Duhem [6] inequality for purely mechanical material models can be simplified to

$$
D=\frac{1}{2} \mathbf{T} \cdot \dot{\mathbf{C}}-\dot{\Psi} \geq 0
$$

In the inequality (1) $D$ denotes the mechanical dissipation, $\mathbf{T}$ the second PiolaKirchhoff stress tensor, $\mathbf{C}=\mathbf{F}^{T} \mathbf{F}$ the right Cauchy-Green tensor, and $\Psi$ the specific free energy. Here $\mathbf{F}$ denotes the deformation gradient with $J=\operatorname{det} \mathbf{F}>0$, and $\mathbf{F}^{T}$ stands for the transpose of F . Following the approach of Naumann and Ihlemann [1], the key idea is to define the free energy for pseudo-hyperelastic material

$$
\Psi\left(W_{0}, W_{\max }\right) \equiv \int_{0}^{W_{0}} \eta\left(\xi, W_{\max }\right) \mathrm{d} \xi
$$

where $W_{0}(\mathbf{C})$ denotes the strain-energy function of the virgin material model (it is assumed that in the undeformed configuration $\left.W_{0}(\mathbf{I})=0\right)$, and

$$
W_{\max }(t) \equiv \max \left\{W_{0}(\tau), \tau \leq t\right\}
$$

The definition directly leads to $\Psi\left(W_{0}(\mathbf{I})=0, W_{\max }\right)=0$, and (after differentiation of (2)) to the interpretation of the softening function

$$
\eta\left(W_{0}, W_{\max }\right)=\frac{\partial \Psi\left(W_{0}, W_{\max }\right)}{\partial W_{0}} .
$$

Inserting the time derivative of $\Psi$ :

$$
\dot{\Psi}=\frac{\partial \Psi\left(W_{0}, W_{\max }\right)}{\partial W_{0}} \dot{W}_{0}+\frac{\partial \Psi\left(W_{0}, W_{\max }\right)}{\partial W_{\max }} \dot{W}_{\max },
$$

and (3) into the inequality (1) we obtain

$$
D=\left(\frac{1}{2} \mathbf{T}-\left.\eta \frac{\partial W_{0}}{\partial \mathbf{C}}\right|_{\mathbf{C}=\mathbf{C}^{T}}\right) \cdot \dot{\mathbf{C}}-\frac{\partial \Psi\left(W_{0}, W_{\max }\right)}{\partial W_{\max }} \dot{W}_{\max } \geq 0 .
$$

This inequality is fulfilled for arbitrary $\dot{\mathbf{C}}$, if the following conditions hold:

$$
\mathbf{T}=\left.2 \eta \frac{\partial W_{0}(\mathbf{C})}{\partial \mathbf{C}}\right|_{\mathbf{C}=\mathbf{C}^{T}}, \quad \frac{\partial \Psi\left(W_{0}, W_{\max }\right)}{\partial W_{\max }} \dot{W}_{\max } \leq 0 .
$$

Due to the definition (3), $W_{\max }$ is always non-negative. Then, a sufficient condition for thermomechanical consistency $(7)_{2}$ is 


$$
\frac{\partial \eta\left(W_{0}, W_{\max }\right)}{\partial W_{\max }} \leq 0
$$

Every function (4) which is a monotonically decreasing function of $W_{\max }$ leads to a possitive dissipation.

Since the Kirchhoff stress tensor is defined as follows

$$
\boldsymbol{\tau} \equiv \boldsymbol{\sigma} J=\mathbf{F T F}^{T},
$$

therefore in the deformed configuration we have

$$
\boldsymbol{\tau}=\left.2 \eta \mathbf{F} \frac{\partial W_{0}(\mathbf{C})}{\partial \mathbf{C}}\right|_{\mathbf{C}=\mathbf{C}^{T}} \mathbf{F}^{T} .
$$

For the softening functions used in $[2,3,7,8]$ :

$$
\eta=1-\frac{1}{c_{2}} \operatorname{Erf}\left[\frac{1}{c_{1}}\left(W_{0}-W_{\max }\right)\right] \quad \text { or } \quad \eta=1-\frac{1}{c_{2}} \operatorname{Tanh}\left[\frac{1}{c_{1}}\left(W_{0}-W_{\max }\right)\right],
$$

where $c_{1}$ and $c_{2}$ are positive constants, the related free energy $\Psi$ (2) of the pseudohyperelastic model can be computed by the integration of (11). These functions lead to a positive dissipation. The free energies related to the softening functions (11) can be given by

$$
\begin{gathered}
\Psi\left(W_{0}, W_{\max }\right)=\frac{c_{1}}{c_{2} \sqrt{\pi}} \operatorname{Exp}\left[-\frac{1}{c_{1}^{2}}\left(\xi-W_{\max }\right)^{2}\right]+\xi-\left.\frac{1}{c_{2}}\left(\xi-W_{\max }\right) \operatorname{Erf}\left[\frac{1}{c_{1}}\left(\xi-W_{\max }\right)\right]\right|_{0} ^{W_{0}} \\
\Psi\left(W_{0}, W_{\max }\right)=\xi+\left.\frac{c_{1}}{c_{2}} \log \left[\operatorname{Cosh}\left[\frac{1}{c_{1}}\left(W_{\max }-\xi\right)\right]\right]\right|_{0} ^{W_{0}} .
\end{gathered}
$$

\section{Pseudo-hyperelastic models of isotropic rubberlike materials}

Consider the simplest class of constitutive isotropic models of rubberlike materials $[13,14]$. We assume the rubberlike materials are incompressible, i.e.

$$
J-1=0 .
$$

Consequently, the potential (2) has to incorporate the constraint (14) with the Lagrange multiplier $p$, being the hydrostatic pressure or the spherical part of the Cauchy stress $\boldsymbol{\sigma}$. In the case of incompressible isotropic materials the stored energy function $\Psi_{0}$ is a function of only two independent invariants of isochoric deformations. The tensor $\mathbf{F}$ is decomposed into the isochoric and volumetric part as follows

$$
\mathbf{F}=J^{1 / 3} \overline{\mathbf{F}}=J^{1 / 3} \mathbf{R} \overline{\mathbf{U}}=J^{1 / 3} \overline{\mathbf{V}} \mathbf{R}, \quad \operatorname{det} \overline{\mathbf{F}}=\operatorname{det} \overline{\mathbf{U}}=\operatorname{det} \overline{\mathbf{V}}=1 .
$$

Further, we introduce 


$$
\mathbf{B}=\mathbf{F F}^{T}, \quad \overline{\mathbf{B}}=\overline{\mathbf{F}} \overline{\mathbf{F}}^{T}, \quad \overline{\mathbf{C}}=\overline{\mathbf{F}}^{T} \overline{\mathbf{F}} .
$$

where B denotes the left Cauchy-Green strain tensor, cf. $[13,14]$ and the references therein. For incompressible isotropic materials the stored energy function has the form,

$$
W_{0}=\bar{W}_{0}(\overline{\mathbf{C}})=\bar{W}_{0}(\overline{\mathbf{B}})=\bar{W}_{0}\left(\bar{I}_{1}, \bar{I}_{2}\right)
$$

where

$$
\bar{I}_{1}=\operatorname{tr} \overline{\mathbf{B}}=\operatorname{tr} \overline{\mathbf{C}}, \quad \bar{I}_{2}=\operatorname{tr} \overline{\mathbf{B}}^{-1}=\operatorname{tr} \overline{\mathbf{C}}^{-1} .
$$

From (10), (14) and (17) we obtain the following constitutive relationship of the pseudo-hyperelasticity in the reference configuration

where

$$
\boldsymbol{\sigma}=-p \mathbf{I}+\eta\left(\bar{\beta}_{1} \overline{\mathbf{B}}+\bar{\beta}_{-1} \overline{\mathbf{B}}^{-1}\right),
$$

$$
\bar{\beta}_{1}=2 \frac{\partial \bar{W}_{0}\left(\bar{I}_{1}, \bar{I}_{2}\right)}{\partial \bar{I}_{1}}, \quad \bar{\beta}_{-1}=-2 \frac{\partial \bar{W}_{0}\left(\bar{I}_{1}, \bar{I}_{2}\right)}{\partial \bar{I}_{2}}
$$

We observe that equations (19) and (20) can be applied to arbitrary stored energy functions describing an isotropic incompressible material. For the details on such functions the reader is referred to $[1-3,6-9,13,14]$.

In the paper [6], following the approach of Ogden and Roxburgh [2,3], we proposed a phenomenological pseudo-hyperelastic model to characterize the Mullins effect in filled rubber. We assumed: the stored energy function in the form

$$
\bar{W}_{0}\left(\bar{I}_{1}, \bar{I}_{2}\right)=\frac{1}{2}\left[a_{1}\left(\bar{I}_{1}-3\right)+\frac{1}{2} a_{2}\left(\bar{I}_{1}^{2}-9\right)+\frac{1}{3} a_{3}\left(\bar{I}_{1}^{3}-27\right)+a_{4}\left(\bar{I}_{2}-3\right)+a_{5}\left(\bar{I}_{1} \bar{I}_{2}-3\right)\right],
$$

where $a_{i}$ are constants, the constitutive relationship is given by Eq. (19) and the softening function is defined by Eq. (11) . It can be proved [13] that the function (21) is a particular case of the polynomial Rivlin form of the stored energy function available in Abaqus [15]. Somone can obtained the pseudo-hyperelastic model with the stored energy function (21) and the softening function (11) $)_{1}$ by using two options : HYPERELASTIC and MULLINS EFECT.

\section{An example}

To illustrate a simple pseudo-elastic model of rubberlike materials we used the stored energy function proposed in our monograph [13]:

$$
\bar{W}_{0}\left(\bar{I}_{1}\right)=\frac{\bar{\mu}_{0}}{2}\left(\bar{I}_{1}-3\right)+\sum_{i=1}^{I} \frac{\mu_{i}}{2 \alpha_{i}}\left(\bar{I}_{1}^{\alpha_{i}}-3^{\alpha_{i}}\right),
$$

where $\bar{\mu}_{0}, \mu_{i}$ and $\alpha_{i} \neq 1(i=1, \ldots, I)$ are positive material parameters. The shear modulus in the undeformed, natural state of the virgin material depends on all material parameters

$$
\mu_{0}=\bar{\mu}_{0}+\sum_{i=1}^{I} \mu_{i} 3^{\alpha_{i}-1} .
$$

For $\mu_{i}=0$ we obtained the neo-Hookean material. 
In view of (19) and (22), the stress-stretch relations are given by:

$$
\boldsymbol{\sigma}=-p \mathbf{I}+\eta \bar{\beta}_{1}\left(\bar{I}_{1}\right) \overline{\mathbf{B}}, \quad \bar{\beta}_{1}\left(\bar{I}_{1}\right)=\bar{\mu}_{0}+\sum_{i=1}^{I} \mu_{i} \bar{I}_{1}^{\alpha_{i}-1}
$$

From the plane stress state, Eq. (24) and $\sigma_{3}=0$, we get

$$
p=\eta\left[\bar{\mu}_{0}+\sum_{i=1}^{I} \mu_{i}\left(\tilde{I}_{1}+\tilde{J}^{-2}\right)^{\alpha_{i}-1}\right] \tilde{J}^{-2} .
$$

Moreover, the constitutive relationship takes the form

$$
\tilde{\boldsymbol{\sigma}}=\eta\left[\bar{\mu}_{0}+\sum_{i=1}^{I} \mu_{i}\left(\tilde{I}_{1}+\tilde{J}^{-2}\right)^{\alpha_{i}-1}\right]\left(-\tilde{J}^{-2} \tilde{\mathbf{I}}+\tilde{\mathbf{B}}\right),
$$

where $\tilde{J}=\operatorname{det} \tilde{\mathbf{F}}, \tilde{\mathbf{F}}$ denotes the plane deformation gradient and $\tilde{I}=\operatorname{tr} \tilde{\mathbf{B}}=\operatorname{tr} \tilde{\mathbf{C}}$. We observe that eq. (26) can also be used for membranes and shells.

Both the uniaxial tension test and biaxial tension test are plane stress tests. For example, we get

$$
\sigma_{1}=\eta\left[\bar{\mu}_{0}+\sum_{i=1}^{I} \mu_{i}\left(\bar{\lambda}_{1}^{2}+\frac{2}{\bar{\lambda}_{1}}\right)^{\alpha_{i}-1}\right]\left(\bar{\lambda}_{1}^{2}-\frac{1}{\bar{\lambda}_{1}}\right),
$$

in the case of a simple uniaxial extension.

The comparison between experimental data [4] and model predictions is depicted in Fig.1. Best-fit parameters are summarized in Table 1. They are calculated by an error minimization procedure which simultaneously utilizes both loading and unloading experimental data. The model with a simplified stored energy function (22) and with two alternative stress softening functions has been used. For both cases good correlation between the theoretical and the experimental data has been observed.

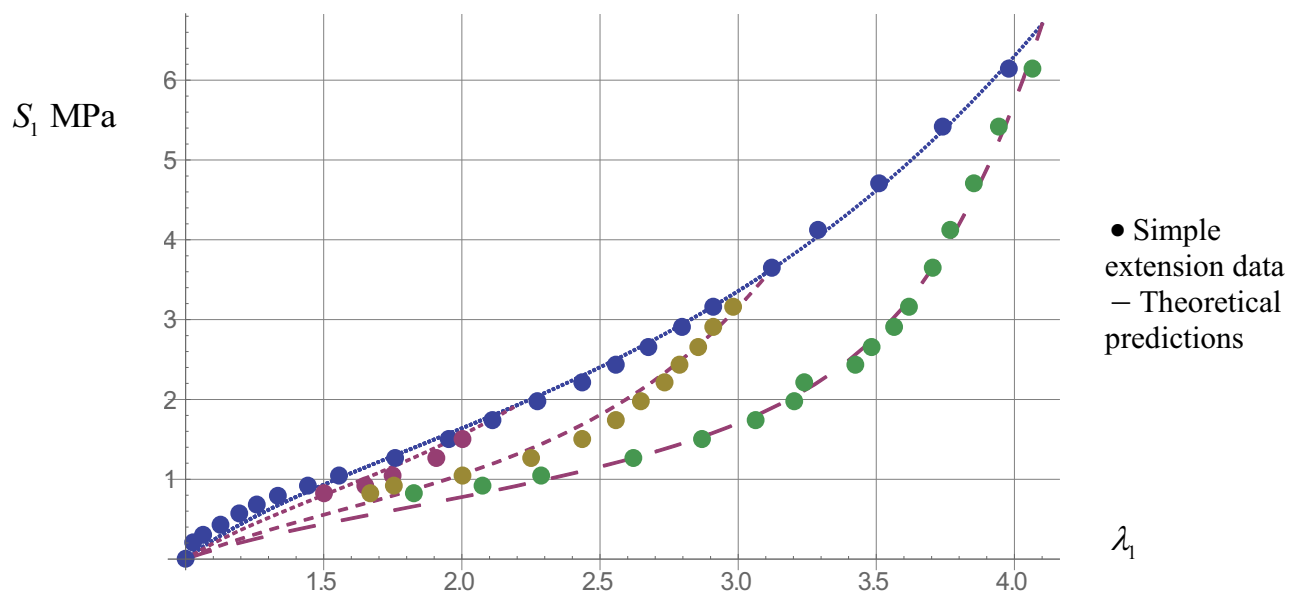

Fig. 1. Nominal stress $S_{1}=\sigma_{1} / \lambda_{1}$ versus stretch $\lambda_{1}$ for loading and unloading uniaxial tension tests. Comparison of theoretical predictions (27) with Mullins and Tobin [4] uniaxial extension data. 
Table 1. Model functions and parameter values

\begin{tabular}{|l|l|}
\hline Energy and softening functions & Value of material parameters \\
\hline $\bar{W}_{0}\left(\bar{I}_{1}\right)=\frac{\bar{\mu}_{0}}{2}\left(\bar{I}_{1}-3\right)+\frac{\bar{\mu}_{1}}{2 \alpha_{1}}\left(\bar{I}_{1}^{\alpha_{1}}-3^{\alpha}\right)$ & $\begin{array}{l}\bar{\mu}_{0}=0.79976 \mathrm{MPa}, \mu_{1}=0.01246 \mathrm{MPa}, \\
\alpha_{1}=2.48471\end{array}$ \\
\hline$\eta=1-\frac{1}{c_{2}} \operatorname{Erf}\left[\frac{1}{c_{1}}\left(W_{0}-W_{\max }\right)\right]$ & $c_{1}=4.18084, c_{2}=1.88581$ \\
$\eta=1-\frac{1}{c_{2}} \operatorname{Tanh}\left[\frac{1}{c_{1}}\left(W_{0}-W_{\max }\right)\right]$ & $c_{1}=3.83054, c_{2}=1.79701$ \\
\hline
\end{tabular}

\section{Final remarks}

The constitutive modelling of isotropic incompressible pseudo-hyperelastic materials can be extended to compressible and anisotropic materials, for instance orthotropic or transversally isotropic. Then in the pseudo-potentials (12) or (13) one has to substitute an appropriate stored energy function similarly, cf. papers [10-12,14].

\section{References}

1. Ch. Naumann, J. Ihlemann, Int. J. Solids Struct. 69-70, 360 (2015)

2. R.W. Ogden, D.G. Roxburg, Proc. R. Soc. London, Ser. A : Math. Phys. Eng. Sci. 455, 2861 (1999)

3. R.W. Ogden, D.G. Roxburgh, Constitutive models for rubber, Dorfman A., Muhr A. (eds), Proc. The First European Conference on Constitutive Models for Rubber, 23 (A.A. Balkema, Roterdam-Brookfield, 1999)

4. L. Mullins, N.R. Tobin, Rubber Chem. Technol. 30, 551 (1957)

5. J. Diani, B. Fayolle, P. Gilormini, Eur. Polymer J. 45, 601 (2009)

6. S. Jemioło, Theoretical Foundations of Civil Enginierings, Rymsza B. [ed], Proc. The $X$ Russian-Polish Semminar, 89 [in Polish] (OW PW, Warszawa 2001)

7. M. Šilhavý, The mechanics and thermodynamics of continuous media (Springer, Berlin - Tokyo 1997)

8. A. Dorfman, R.W. Ogden, Int. J. Solids Struct., 41, 1855 (2004)

9. S. Jemioło, J.J. Telega, Acta Bioeng. Biomech. 1 (Suppl. 1), 207 (1999)

10. S. Jemioło, J.J. Telega, Eng. Trans., 49(2-3), 213 (2001)

11. S. Jemioło, J.J. Telega, Acta Bioeng. Biomech. 3 (Suppl. 2), 209 (2001)

12. S. Jemioło, J.J. Telega, C. Michalak, Acta Bioeng. Biomech. 4 (Supp.1), 470 (2002)

13. S. Jemioło, Study of hyperelastic properties of isotropic materials. Modeling and numerical implementation. Scientific Works. Civil Engineering 140 [in Polish] (OWPW, Warszawa, 2002)

14. S. Jemioło, Constitutive relationships of hyperelasticity [in Polish], (PAN, KILiW, Warszawa, 2016)

15. Abaqus v. 6.12, Theory Manual (Simulia, 2012) 